\title{
Aspectos associados à drogarresistência em pessoas com Tuberculose/HIV: revisão integrativa
}

Aspects associated with drug resistance in people with tuberculosis/HIV: an integrative review Aspectos relacionados con la drogorresistencia en personas con tuberculosis/VIH: revisión integradora

Vanessa da Frota Santos ${ }^{1}$ iD https://orcid.org/0000-0002-1198-6560

Ana Karoline Bastos Costa ${ }^{1}$ io https://orcid.org/0000-0002-5994-081X

Ivana Cristina Vieira de Lima ${ }^{1}$ is https://orcid.org/0000-0002-2698-9086 Marli Teresinha Gimeniz Galvão ${ }^{1}$ io https://orcid.org/0000-0003-3995-9107

Como citar:

Santos VF, Costa AK, Lima IC, Galvão MT. Aspectos associados à drogarresistência em pessoas com Tuberculose/HIV: revisão integrativa. Acta Paul Enferm. 2020;33:AAPE20190131.

DOI

http://dx.doi.org/10.37689/actaape/2020AR01316

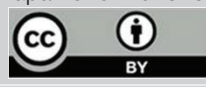

Descritores

HIV: Tuberculose; Coinfeccões por HIV: Síndrome de Imunodeficiência Adquirida; Resistência a múltiplos medicamentos

Keywords

VIH: Tuberculosis; VIH infections; Acquired immunodeficiency syndrome; Drug resistance, multiple

Descriptores VIH; Tuberculose; Infecciones por VIH; Síndrome de Inmunodeficiencia Adquirida; resistencia a múltiples medicamentos

\section{Submetido}

21 de Maio de 2019

Aceito

3 de Março de 2020

Autor correspondente

Vanessa da Frota Santos

Email: doutorandavanessasantos@outlook.

\section{Resumo}

Objetivo: Analisar as produções científicas acerca dos aspectos associados à drogarresistência em pessoas com coinfecção Tuberculose/HIV.

Métodos: Revisão integrativa de literatura, realizada nas bases de dados CINAHL, LILACS, SciELO, Web of Science, SCOPUS, MEDLINE e COCHRANE. Utilizaram-se dos descritores HIV, Tuberculose e Resistência a múltiplos medicamentos, em português, inglês e espanhol. Encontraram-se 1.177 artigos e selecionaram-se 19, excluíram-se 1.158, 41 duplicados e 1.117, por não atenderem à pergunta de pesquisa e abordarem outras temáticas, como: coinfecção do HIV e pneumonia; impacto da multirresistência na vida de pessoas com coinfecção, destacando-se a mortalidade; testes diagnósticos de multirresistência aos fármacos; e associação entre HIV e tuberculose meníngea.

Resultados: Os dados obtidos foram organizados em três categorias temáticas: Aspectos clínicos, destacandose: infecção pelo HIV, hipoalbuminemia, carga elevada do bacilo; Aspectos relacionados aos fármacos, incluindo abandono do tratamento, não adesão à terapia, tratamento prévio para tuberculose, má absorção de medicamentos, efeitos adversos causados pela terapia antirretroviral, interação entre os tratamentos de ambas as infecções; e Aspectos sociais, sinalizando-se hospitalizações, convivência com outras pessoas com bacilos multirresistentes, privação de liberdade, atraso no diagnóstico e início tardio do tratamento.

Conclusão: Os principais aspectos identificados foram 0 abandono da terapêutica, tratamento prévio para tuberculose e intervenção inadequada, tais resultados, também, podem estender-se às pessoas que não apresentam coinfecção. Enfatiza-se a importância desta revisão para instigar novas pesquisas, com destaque para estratégias com foco na identificação precoce de pessoas com multirresistência, prevenção e incentivo à adesão ao tratamento.

\section{Abstract}

Objective: To analyze scientific productions on aspects associated with drug resistance in people with tuberculosis (TB)/HIV coinfection.

Methods: Integrative literature review performed in the CINAHL, LILACS, SciELO, Web of Science, SCOPUS, MEDLINE and COCHRANE databases. The following descriptors were used in Portuguese, English and Spanish: HIV, Tuberculosis and Multidrug resistance. In total, 1,177 articles were found and 19 were selected; 1,158 were excluded, of which 41 were duplicates and 1,117 did not answer the research question and addressed other topics, namely: HIV and pneumonia coinfection; impact of multidrug resistance on the lives of people with coinfection, with emphasis on mortality; diagnostic tests for multidrug resistance; and association between HIV and meningeal tuberculosis. 
Results: Data were organized into three thematic categories, as follows: Clinical aspects, highlighting: HIV infection, hypoalbuminemia, elevated bacillus load; drug-related aspects, including treatment abandonment, non-adherence to therapy, previous treatment for tuberculosis, drug malabsorption, adverse effects caused by antiretroviral therapy, interaction between treatments for both infections; and social aspects, including hospitalizations, living with other people with multidrug-resistant bacilli, deprivation of liberty, delayed diagnosis and late start of treatment.

Conclusion: The main aspects identified were abandonment of therapy, previous treatment for tuberculosis and inadequate intervention, and these results may also extend to people who do not have coinfection. This review is important to instigate new research, with emphasis on strategies focused on the early identification of people with multidrug resistance, prevention and encouragement of adherence to treatment.

\section{Resumen}

Objetivo: Analizar las producciones científicas sobre los aspectos relacionados con la drogorresistencia en personas con coinfección tuberculosis/VIH.

Métodos: Revisión integradora de literatura, realizada en las bases de datos CINAHL, LILACS, SciELO, Web of Science, SCOPUS, MEDLINE y COCHRANE. Se utilizaron los descriptores VIH, tuberculosis y resistencia a múltiples medicamentos, en portugués, inglés y español. Se encontraron 1.177 artículos, de los que se seleccionaron 19 y se excluyeron 1.158, 41 duplicados y 1.117 por no abordar la pregunta de la investigación y tratar otros temas, como: coinfección de VIH y neumonía; impacto de la multirresistencia en la vida de personas con coinfección, con énfasis en la mortalidad; pruebas diagnósticas de multirresistencia a los fármacos, y relación entre VIH y tuberculosis meníngea.

Resultados: Los datos obtenidos fueron organizados en tres categorías temáticas: aspectos clínicos, con énfasis en: infección por VIH, hipoalbuminemia, carga del bacilo elevada; aspectos relacionados con los fármacos, como abandono del tratamiento, no adherencia al tratamiento, tratamiento previo para tuberculosis, mala absorción de medicamentos, efectos adversos causados por el tratamiento antirretroviral, interacción entre los tratamientos de ambas infecciones; y aspectos sociales, con foco en internaciones, convivencia con otras personas con bacilos multirresistentes, privación de la libertad, retraso en el diagnóstico e inicio tardío del tratamiento.

Conclusión: Los principales aspectos identificados fueron el abandono del tratamiento, el tratamiento previo para tuberculosis y la intervención inadecuada. Estos resultados también pueden extenderse a las personas que no presentan coinfección. Se resalta la importancia de esta revisión para estimular nuevas investigaciones, con énfasis en estrategias centradas en la identificación temprana de personas con multirresistencia, prevención e incentivo para adherir al tratamiento.

\section{Introdução}

A Tuberculose (TB) está entre as infecçóes mais frequentes em pessoas com Vírus da Imunodeficiência Humana (HIV), ${ }^{(1)}$ sendo difícil de ser diagnosticada, em virtude da própria imunodeficiência, que acarreta a possibilidade de modificação da apresentação radiológica e clínica, bem como a menor sensibilidade à baciloscopia. A TB pode acometer a pessoas com HIV em diferentes estágios da doença, embora seja mais incidente naqueles com supressão imunológica, ocasionando maior risco de progressão para o estágio final da doença e de morte. ${ }^{(2)}$

Ambas as infecçóes compartilham fatores de risco sociodemográficos, com destaque para maior prevalência entre as pessoas com menor grau de escolaridade e renda. Além disso, são consideradas condiçôes crônicas de difícil controle e adesão aos fármacos, em decorrência da complexidade do tratamento e dos efeitos colaterais. ${ }^{(3)}$

Estima-se que 10 milhóes de pessoas desenvolveram a TB no mundo, em 2017, e houve estimativa de 1,3 milhóes de mortes, sendo 300.000 associadas ao HIV. No referido ano, mundialmente, desses 10 milhóes de pessoas, 9\% eram coinfectadas com o HIV. ${ }^{(4)}$ No Brasil, dos 69.509 casos novos de TB notificados, em 2016, 6.501 apresentaram resultado positivo para HIV, o que representa proporção de coinfecção por Tuberculose/HIV (TB/HIV) de 9,4\%. ${ }^{(5)}$ A associação entre HIV e TB tem se tornado desafio tanto para o diagnóstico quanto para o tratamento, acarretando aumento da mortalidade, principalmente quando associada à multirresistência aos fármacos. ${ }^{(6)}$

Em 2016, diagnosticaram-se, no Brasil, 1.044 casos com resistência a medicamentos para $\mathrm{TB}$, sendo inserido no Sistema de Informação de Tratamentos Especiais de Tuberculose (SITETB) 752 casos novos de TB drogarresistente, considerando 177 (23,5\%) monorresistentes, 330 (43,9\%) de resistência à rifampicina, 49 (6,5\%) polirresistentes, $193(25,7 \%)$ de multirresistência e três $(0,3 \%)$ não tinham informação. ${ }^{(5)}$

Existem duas formas de desenvolvimento da TB drogarresistente, a primária é a adquirida, verifica$\mathrm{da}$ em pessoas nunca tratadas para TB que foram contaminadas por bacilos previamente resistentes. A segunda é a resistência adquirida ou secundária, encontrada em pessoas com TB inicialmente sensível e que se tornam resistentes após exposição aos medicamentos, tendo como causas inúmeros fatores, dentre os quais se destacam: abandono de tra- 
tamento; esquemas inadequados, que acarretaram seleção de cepas resistentes e mutantes; baixa adesão à terapia, ampla gama de doenças sistêmicas e tratamentos com imunossupressores. ${ }^{(7)}$

Dentre outros aspectos considerados preditores para o desenvolvimento da TB drogarresistente, apontam-se os pessoais, como o uso de álcool e a própria infecção pelo HIV. A multirresistência dificulta o controle, a prevenção e o tratamento da doença, o que pode ocasionar a piora do quadro clínico da pessoa, aumentando, assim, os riscos de morbidade e mortalidade. ${ }^{(1)}$

Diante disso, a Organização Mundial de Saúde (OMS) lançou, como objetivo global, eliminar a endemia da $\mathrm{TB}$, reduzir em $90 \%$ o número de mortes e $80 \%$ das taxas de incidência até 2030 , de modo a erradicar a doença até 2050. Além de lançar estratégias que objetivam apoiar essas metas, como Fim da TB (End TB Strategy). ${ }^{(2)}$

Compreender os principais aspectos que motivam a multirresistência em pessoas com coinfecção TB/HIV é fundamental para o delineamento de ações de prevenção, identificação e manejo adequado dessa condição, com vistas a minimizar mortes evitáveis. Assim, objetivou-se analisar as produçóes científicas acerca dos aspectos associados à drogarresistência em pessoas com coinfecção TB/HIV.

\section{Métodos}

Trata-se de revisão integrativa da literatura, desenvolvida a partir das seguintes etapas: elaboração da pergunta norteadora, definição dos critérios de inclusão e exclusão das produçóes científicas, busca nas bases de dados, análise dos resumos dos estudos, seleção dos estudos, avaliação criteriosa dos estudos selecionados e análise dos dados ${ }^{(8)}$ e das recomendaçóes previstas no PRISMA Statement. ${ }^{(9)}$

Buscou-se responder à questão norteadora, formulada com base na estratégia PICO: ${ }^{(10)}$ quais os aspectos associados à drogarresistência em pessoas com coinfecção TB/HIV? Os critérios de inclusão consistiram em artigos completos, disponíveis eletronicamente, nos idiomas português, inglês e espanhol, sem recorte temporal. Excluíram-se publicaçôes repetidas, as quais foram agrupadas na base de dados que continha mais artigos, aquelas que não responderam à questão de pesquisa, além de estudos com crianças e animais.

A busca eletrônica foi realizada por dois revisores, de forma simultânea, durante os meses de julho e agosto de 2018, em quatro bases de dados: Cumulative Index to Nursing and Allied Health Literature (CINAHL), Literatura LatinoAmericana e do Caribe em Ciências da Saúde (LILACS/BIREME), Web of Science e SCOPUS, um portal: Medical Literature Analysis and Retrieval System Online (MEDLINE/Pubmed) e duas bibliotecas: Scientific Eletronic Library Online (SciELO) e COCHRANE. Utilizaram-se dos descritores "HIV", "Tuberculose" e "Resistência a múltiplos medicamentos", disponíveis no Medical Subject Headings (MeSH) e no Descritores em Ciências da Saúde (DeCS), nos idiomas português, inglês e espanhol, com uso do operador booleano $A N D$.

A partir do cruzamento, foram identificadas 1.177 publicaçóes, sendo selecionados 19 artigos, os quais foram 17 indexados na MEDLINE e dois na SCOPUS. Eliminaram-se 1.158 artigos, dentre os quais 41 estavam duplicados e 1.117 não atendiam à questão norteadora, por abordarem as seguintes temáticas: coinfecção do HIV e pneumonia; impacto da multirresistência na vida de pessoas com coinfecção, destacando-se a mortalidade; testes diagnósticos de multirresistência aos fármacos; associação entre HIV e TB meníngea.

A figura 1 mostra a identificação, seleção e inclusão dos artigos nesta revisão.

A análise dos dados foi realizada mediante tradução e leitura dos artigos na íntegra, por dois autores, de forma independente. Em caso de dúvidas, houve reunião entre os revisores para consenso. As informações foram transcritas e organizadas, a partir de instrumento validado, ${ }^{(11)}$ que investigou: autoria, ano, país de publicação, tipo de estudo, aspectos associados à drogarresistência em pessoas com TB/ HIV e os níveis de evidência.

Os níveis de evidência foram determinados da seguinte forma: Nível I -Evidências provenientes de revisão sistemática ou metanálise de múltiplos estudos clínicos controlados e randomizados ou oriundos de diretrizes clínicas, baseadas em revisóes sistemáticas de 


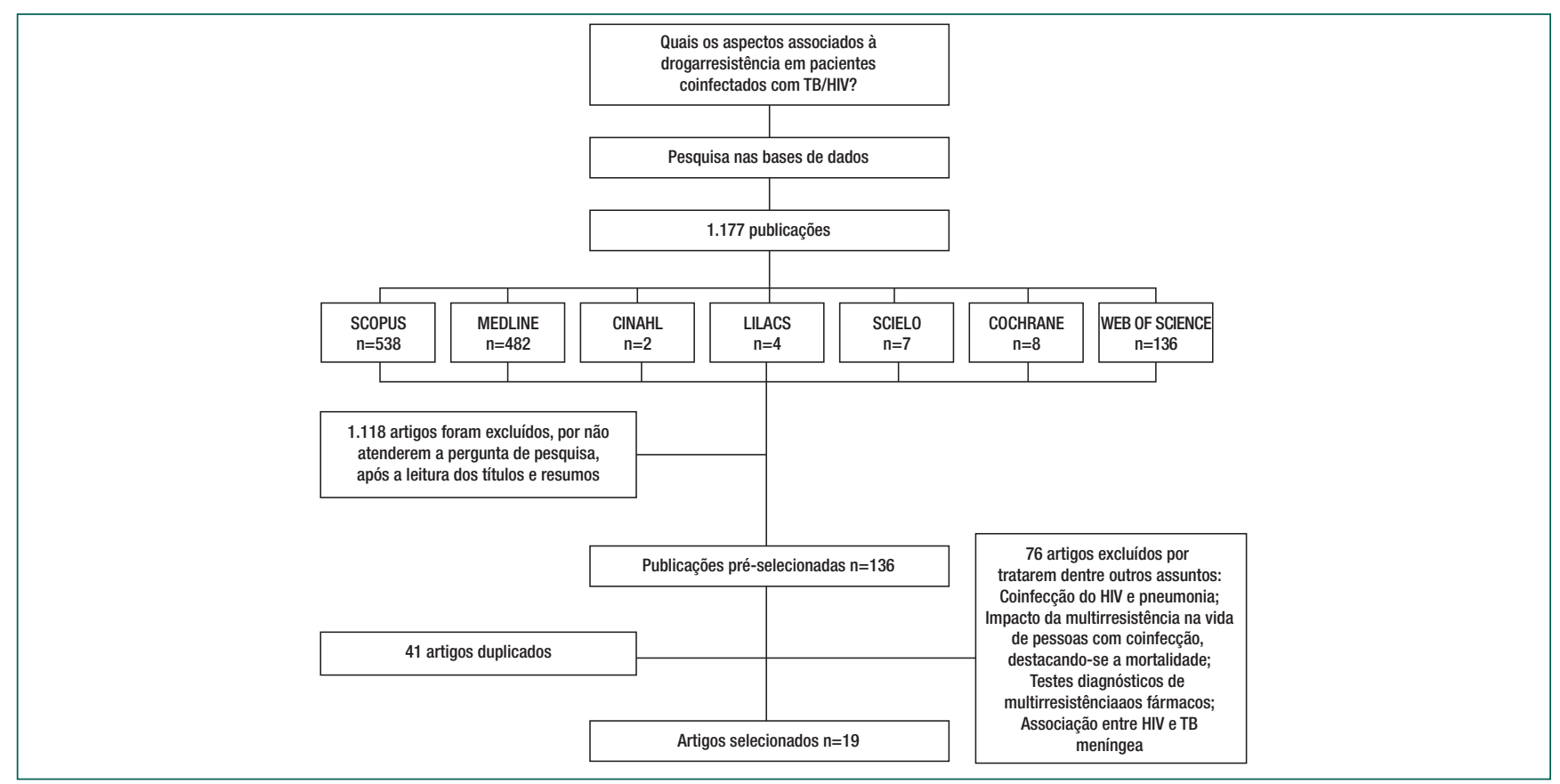

Figura 1. Fluxograma de identificação, seleção e inclusão dos estudos

ensaios clínicos controlados e randomizados; Nível II -Evidências provenientes de estudos individuais controlados e randomizados; Nível III -Evidências de estudos experimentais sem randomização; Nível IV -Evidências provenientes de coorte ou caso-controle; Nível V -Evidências oriundas de revisão sistemática de estudos descritivos e qualitativos; Nível VI -Evidências originárias de estudo descritivo ou qualitativo; Nível VII -Evidências obtidas de opinióes de autoridades ou relatório de comitês de especialistas. ${ }^{(12)}$

Após análise, os aspectos associados à drogarresistência em pessoas com coinfecção TB/HIV foram agrupados em três categorias, delimitadas de acordo com os achados dos estudos: 1. Aspectos clínicos, 2. Aspectos relacionados aos fármacos e 3. Aspectos sociais. Os achados foram discutidos com embasamento na literatura científica, sendo respeitada a integridade dos artigos e os direitos autorais, náo havendo modificação do conteúdo encontrado em benefício desta pesquisa.

\section{Resultados}

Quanto à caracterização dos estudos, o ano de publicação variou de 1992 a 2017, sendo oito publicados em países europeus, 10 nos Estados Unidos e um no continente africano. A análise dos níveis de evidência mostrou a seguinte distribuição: um nível II; ${ }^{(13)}$ três artigos classificados no nível IV; ${ }^{(14-16)}$ cinco, nível $\mathrm{V}^{(17-21)}$ e dez artigos, nível VI. ${ }^{(22-31)}$ Houve destaque para os estudos descritivos e transversais (Quadro 1).

Os aspectos relacionados à drogarresistência em pessoas com coinfecção TB/HIV foram classificados em uma das três categorias: a) Aspectos clínicos, b) Aspectos relacionados aos fármacos e c) Aspectos sociais. A categoria 1 abordou os aspectos clínicos que influenciam o aumento da drogarresistência aos fármacos em pessoas com coinfecção TB/HIV, destacando-se a própria infecção pelo HIV que está fortemente relacionada ao desenvolvimento da multirresistência aos fármacos para o tratamento da TB em pessoas com coinfecção. Outro aspecto relacionado foi a hipoalbuminemia, associada à desnutrição, podendo prejudicar a imunidade do hospedeiro com Mycobacterium tuberculosis. Além disso, tem-se a carga elevada do bacilo que pode ser considerada causa da resistência aos fármacos, visto que a alta carga bacteriana no organismo pode estar relacionada com a dificuldade no tratamento (Quadro 1).

A categoria 2 referiu-se aos aspectos que se relacionam aos fármacos, destacando-se o abandono do tratamento para TB como fundamental para o desenvolvimento da drogarresistência em pessoas com 
coinfecção TB/HIV, além da não adesão à terapia, tratamento inadequado e falhas no tratamento para TB. Outro fator referido foi o tratamento prévio para TB que tem relação direta com o desenvolvimento da multirresistência, além da má absorção de medicamentos e os efeitos adversos causados pela terapia antirretroviral. Outro aspecto identificado foi a interação entre os tratamentos de ambas as infecções, acarretando a redução da concentração sanguínea do medicamento antituberculose, quando associado ao antirretroviral (Quadro 1).
Com relação à categoria 3, destacaram-se as hospitalizaçóes em locais com pessoas com HIV, a convivência com outras que apresentavam bacilos multirresistentes ou TB ativa, em ambientes hospitalares, bem como o contato em salas de espera para consultas com essas pessoas, o que aumenta o risco de transmissão das cepas resistentes; e a privação de liberdade e a consequente permanência dessas pessoas em presídios. Além disso, o atraso no diagnóstico e o consequente início tardio do tratamento da TB também foram considerados aspectos preditores da resistência aos fármacos (Quadro 1).

Quadro 1. Caracterização dos artigos selecionados quanto à autoria, ao ano de publicação, país de publicação, tipo de estudo, aspectos associados à drogarresistência em pessoas coinfectadas com TB/HIV e nível de evidência

\begin{tabular}{|c|c|c|c|c|}
\hline $\begin{array}{l}\text { Autor/ } \\
\text { Ano }\end{array}$ & País de publicação & Tipo de estudo & $\begin{array}{l}\text { Aspectos associados à drogarresistência em pessoas com } \\
\text { coinfecção TB/HIV }\end{array}$ & $\begin{array}{l}\text { Nivel de } \\
\text { Evidência* }\end{array}$ \\
\hline Fischl et al., 1992 $2^{(14)}$ & Estados Unidos & Caso-controle & $\begin{array}{l}\text { Infecção pelo HIV; } \\
\text { Hospitalizações; } \\
\text { Exposição a outras pessoas com TB multirresistente. }\end{array}$ & IV \\
\hline Sacks et al., 1999(22) & Estados Unidos & Descritivo Prospectivo & $\begin{array}{l}\text { Infecção pelo HIV; } \\
\text { Tratamento prévio para TB. }\end{array}$ & VI \\
\hline Campos et al., 2003(15) & Estados Unidos & Caso-controle & $\begin{array}{l}\text { Tratamento inadequado; } \\
\text { Baixa adesão ao tratamento; } \\
\text { Infecção pelo HIV. }\end{array}$ & VI \\
\hline Aaron et al., 2004 & Inglaterra & Revisão de literatura & $\begin{array}{l}\text { Infecção pelo HIV; } \\
\text { Má absorção dos fármacos; } \\
\text { Abandono do tratamento. }\end{array}$ & V \\
\hline Drobniewski et al., 2005(25) & Inglaterra & Transversal & Tratamento inadequado & $\mathrm{VI}$ \\
\hline Kawai et al., 2006 & Estados Unidos & Descritivo e longitudinal & $\begin{array}{l}\text { Tratamento prévio para TB; } \\
\text { Infecção pelo HIV; } \\
\text { Não disponibilidade de testes de susceptibilidade aos bacilos } \\
\text { multirresistentes. }\end{array}$ & IV \\
\hline Vermund; yamamoto, 2007(21) & Escócia & Revisão de literatura & $\begin{array}{l}\text { Abandono do tratamento; } \\
\text { Tratamento inadequado. }\end{array}$ & V \\
\hline Kim et al., 2007 ${ }^{(28)}$ & Estados Unidos & Transversal & $\begin{array}{l}\text { Hipoalbuminemia; } \\
\text { Desnutrição. }\end{array}$ & VI \\
\hline Bifani et al., 2008 ${ }^{233}$ & Estados Unidos & Transversal & Infecção pelo HIV & $\mathrm{VI}$ \\
\hline Chakraborty et al., 2010 & Inglaterra & Transversal & $\begin{array}{l}\text { Uso excessivo de medicamentos de primeira linha; } \\
\text { Abandono do tratamento. }\end{array}$ & $\mathrm{VI}$ \\
\hline Hom et al., 2012(27) & Estados Unidos & Transversal & Infecção pelo HIV & $\mathrm{VI}$ \\
\hline Munawwar; singh, 2012 & Escócia & Revisão sistemática & $\begin{array}{l}\text { Fatores associados ao HIV; } \\
\text { Exposição a outras pessoas com TB; } \\
\text { Aumento dos efeitos adversos da TARV; } \\
\text { Má absorção dos medicamentos com fraca farmacocinética; } \\
\text { Desnutrição; } \\
\text { Atraso no diagnóstico. }\end{array}$ & V \\
\hline Janbaz et al., 2012 $2^{(19)}$ & Inglaterra & Revisão de literatura & Abandono do tratamento & V \\
\hline Mclleron et al., 2012(29) & Estados Unidos & Descritivo longitudinal & Tratamento inadequado & $\mathrm{VI}$ \\
\hline Berhan; berhan; yizengaw, 2013(18) & Etiópia & $\begin{array}{l}\text { Metanálise de estudos } \\
\text { longitudinais e transversais }\end{array}$ & Tratamento prévio para TB & V \\
\hline Kock et al., 2014 & Estados Unidos & $\begin{array}{l}\text { Ensaio clínico randomizado } \\
\text { crossover }\end{array}$ & Interação entre os medicamentos antituberculose e antirretrovirais & $\|$ \\
\hline Elmi et al., 2015 $5^{(16)}$ & Itália & Caso-controle & $\begin{array}{l}\text { Baixa adesão ao tratamento; } \\
\text { Abandono do tratamento; } \\
\text { Infecção pelo HIV; } \\
\text { Tratamento prévio para TB; } \\
\text { Carga bacteriana elevada; } \\
\text { Exposição a outras pessoas com TB multirresistente. }\end{array}$ & IV \\
\hline Heysell et al., 2016 (26) & França & Transversal & Abandono do tratamento & $\mathrm{VI}$ \\
\hline Rockwood et al., 2017(31) & Estados Unidos & Transversal & Privação de liberdade & VI \\
\hline
\end{tabular}

* Nível I -Evidências provenientes de revisão sistemática ou metanálise de múltiplos estudos clínicos controlados e randomizados ou oriundos de diretrizes clínicas, baseadas em revisões sistemáticas de ensaios clínicos controlados e randomizados; Nível II -Evidências provenientes de estudos individuais controlados e randomizados; Nível III -Evidências de estudos experimentais sem randomização; Nível IV -Evidências provenientes de coorte ou caso-controle; Nível V -Evidências oriundas de revisão sistemática de estudos descritivos e qualitativos; Nível VI -Evidências originárias de um estudo descritivo ou qualitativo; Nível VII -Evidências obtidas de opiniões de autoridades ou relatório de comitês de especialistas 


\section{Discussão}

Observou-se que a infecção pelo HIV está fortemente relacionada ao desenvolvimento da multirresistência aos fármacos para tratamento da $\mathrm{TB}$ em pessoas com coinfecção, destacando-se a imunossupressão como um dos fatores que influenciam nesse processo. ${ }^{(23)}$ Outro fator relacionado é a desnutrição, causada pela ingestão de baixas calorias, náuseas frequentes, vômitos e diarreia, associada à TARV. ${ }^{(20)}$ Isso pode prejudicar a imunidade do hospedeiro com Mycobacterium tuberculosis, mediante diminuição da produção de citoquinas, incluindo IFN-g, ou da redução de células T CD4+ e T CD8+, além de estar relacionada às falhas no tratamento de pessoas coinfectadas pela TB/HIV. ${ }^{(28)}$

Além disso, carga elevada do Mycobacterium tuberculosis pode ser considerada causa da resistência aos fármacos. Essa alta carga bacteriana no organismo pode estar relacionada à dificuldade no tratamento, acarretando permanência da positividade bacteriana no esfregaço de Bacilo Álcool-Ácido Resistente (BAAR) e aumento da resistência aos fármacos. Essa relação é muito mais potente em pessoas com tratamento anterior para $\mathrm{TB}$, quando comparada às pessoas em tratamento primário. ${ }^{(16)}$

O abandono do tratamento para TB é outro fator fundamental para o desenvolvimento da resistência aos fármacos em pessoas com coinfecção, podendo ser ocasionada pelo tratamento duplo, acarretando maior propensão ao abandono da terapia, devido à alta carga de drogas e toxicidade ou efeitos colaterais dos medicamentos. ${ }^{(26)}$ Estudo realizado no Marrocos identificou que de um total de 2.532 pessoas tratadas para TB, $10 \%$ destas produziram reaçóes adversas, sendo prevalentes as gastrointestinais $(7,4 \%)$, seguidas das cutâneas $(3,7 \%)$, hepáticas $(2,0 \%)$, articulares $(1,14 \%)$, imunoalergênicas $(1,07 \%)$, neuropsiquiátricas $(0,7 \%)$ e, em menor incidência, as oculares $(0,1 \%){ }^{(32)}$

As reaçóes adversas a medicamentos representam obstáculo potencial para conclusão do tratamento e podem afetar negativamente o resultado esperado. A documentação, avaliação, gestão e intervenção imediata sobre os sintomas das reações adversas a medicamentos é importante para alcançar melhor adesão e melhorar os resultados do tratamento. ${ }^{(33)}$
Em metanálise realizada, observou-se que a taxa de sucesso do tratamento para TB foi significativamente menor entre as pessoas com HIV (67\%), quando comparada com os casos que não apresentavam o vírus (81\%). Além disso, identificou-se que das pessoas que não apresentaram sucesso no tratamento antituberculose, quase $50 \%$ tiveram morte como desfecho. ${ }^{(34)}$

Assim, devem-se desenvolver medidas que busquem reduzir as taxas de interrupção do tratamento, mediante fornecimento de apoio social e psicológico às pessoas mais vulneráveis, além de outras estratégias, como ajuda de custo em passagens para locomoção e fornecimento de alimentos, em virtude dos efeitos ocasionados pela terapia farmacológica. ${ }^{(26)}$ Também, é importante fortalecer os serviços de atendimento de pessoas com $\mathrm{TB} / \mathrm{HIV}$, mediante medidas como o Tratamento Diretamente Observado (TDO), por profissionais especializados. ${ }^{(18)}$

A não adesão ao tratamento, também, foi citada como causa do desenvolvimento da resistência às drogas antituberculose, devido à tomada pouco frequente e irregular das medicaçóes. ${ }^{(16)}$ Em estudo realizado na Etiópia, verificou-se que residir distante da capital, ter baixo peso no início do tratamento da TB, estar na condição de acamado e ter experimentado algum efeito colateral das medicaçóes antituberculose foram fatores que resultaram em falhas no tratamento. ${ }^{(6)}$

Outra causa foi o tratamento inadequado, com prescriçôes fixas de rifampicina, isoniazida, pirazinamida e etambutol (medicamentos de primeira linha), preconizadas pela OMS, de acordo com o peso do paciente. Porém, essas concentraçóes não foram avaliadas em todas as faixas de peso, o que pode ocasionar subdosagem, resultando em infecção prolongada, falhas no tratamento e multirresistência. ${ }^{(15,25,29)}$ A administração de doses inadequadas acarretam o uso aumentado de drogas de primeira linha, sem avaliação inicial e individual de cada paciente, em relação à concentração ideal do fármaco, ocasionando o aumento das chances de desenvolvimento de multirresistência. ${ }^{(24)}$

Ademais, motivo que merece destaque é o tratamento prévio para TB que tem relaçáo direta com o desenvolvimento da multirresistência. ${ }^{(30)}$ Pesquisa mundial, realizada pela OMS, no período de 20022007, mostrou que a prevalência de TB multirresistente foi de $60 \%$ entre os casos tratados anteriormente. ${ }^{(18)}$ 
Destacou-se, também, a má absorção de medicamentos e a consequente diminuição da ação farmacocinética que estão associadoas à imunossupressão ocasionada pelo HIV. ${ }^{(17,20)}$ Além disso, os efeitos adversos causados pela terapia antirretroviral podem ocasionar a multirresistência aos medicamentos para tratamento da TB. ${ }^{(20)}$

Acrescentam-se, também, as hospitalizações em locais com pessoas vivendo com $\mathrm{HIV}^{(14)}$ e o contato com outras pessoas com bacilos multirresistentes ou TB ativa, preditores para o desenvolvimento da drogarresistência. Isso pode ser justificado pelas visitas constantes aos centros de atendimento para pessoas com coinfecção TB/HIV e pelo contato em salas de espera para consultas com essas pessoas, o que aumenta o risco de transmissão das cepas resistentes. ${ }^{(14,20)}$ Ademais, o tempo de permanência em instituições com privação de liberdade aumenta o risco de desenvolvimento da resistência aos fármacos antituberculose em homens coinfectados, uma vez que os sistemas de saúde são precários nessas instituiçóes, há o aglomerado de pessoas e o ambiente pouco ventilado, aumentando o risco de disseminação do bacilo, além do preconceito e do medo de revelação do diagnóstico, o que acarreta interrupçôes do tratamento, tanto da TB como do HIV, durante o encarceramento. ${ }^{(31)}$

$\mathrm{O}$ atraso no diagnóstico da TB drogarresistente e o consequente início tardio do tratamento, também, foram considerados fatores preditores da resistência aos fármacos. Isso pode ser ocasionado pela não realização precoce de testes de susceptibilidade aos bacilos multirresistentes, o que ocasiona o uso de medicamentos de primeira linha durante os primeiros seis ou nove meses, para depois identificar a resistência e iniciar a terapia adequada com fármacos de segunda linha, mais tóxicos e dispendiosos, gerando falhas no tratamento e acarretando riscos para saúde da pessoa, como aumento da mortalidade e da infecção prolongada pela TBMDR. ${ }^{(20)}$

Para se evitar tais condutas, são necessárias ações como: sensibilização e capacitação da equipe multiprofissional quanto ao diagnóstico, tratamento e adesão à terapêutica; realização do teste para sorologia do HIV em todas as pessoas com diagnóstico de TB; notificação e alimentaçáo adequada dos sistemas de informação. ${ }^{(35)}$ Por fim, ressalta-se a importância da criação de estratégias e do envolvimento político de gestores e profissionais de saúde para melhoria da qualidade de vida e aumento da sobrevida dessa população.

\section{Conclusão}

Evidenciou-se que vários aspectos podem ser preditores para o desenvolvimento da resistência aos fármacos antituberculose, em pessoas com coinfecção TB/HIV, contemplando aspectos clínicos, relacionados aos fármacos e sociais, acarretando prejuízos na saúde dessa população e mudanças no curso da coinfecção. Constatou-se, também, que a maioria dos aspectos foram os relacionados aos fármacos, de forma que as variáveis mais encontradas foram: abandono da terapêutica, tratamento prévio para TB e intervenção inadequada. Enfatiza-se a importância dessa revisão para identificação dos principais aspectos associados à resistência aos fármacos em pessoas com coinfecção TB/HIV, instigando novas pesquisas, com destaque para necessidade de investigar estratégias educativas, com foco na identificação precoce de pessoas que apresentem multirresistência, prevenção e incentivo à adesão ao tratamento, com vistas à reduçáo das taxas de abandono da terapia e de mortalidade. Aponta-se a necessidade de sensibilizaçáo dos profissionais de saúde sobre a importância da prescrição adequada do tratamento e a redução do uso indiscriminado de medicamentos antituberculose, buscando promover a saúde e melhorar a condição clínica dessas pessoas, incluindo estratégias amplas de prevenção. Além de ações de capacitação de profissionais, nos serviços de saúde, que atendem a esse público, sobre a conduta adequada de cada caso e da importância do trabalho interdisciplinar e multiprofissional, buscando promover a construçáo do cuidado integral e articulado, envolvendo ambos os programas das infecçóes.

\section{Referências}

1. Fernandez D, Salami I, Davis J, Mbah F, Kazeem A, Ash A, et al. HIV-TB Coinfection among 57 million pregnant women, obstetric complications, alcohol use, drug abuse, and depression. J Pregnancy. 2018;2018:5896901.

2. World Health Organization (WHO). WHO End TB Strategy: global strategy and targets for tuberculosis prevention, care and control after 2015 [Internet]. Genebra: WHO; 2015 [cited 2018 Mar 1]. Available from: https://www.who.int/tb/post2015_strategy/en/ 
3. Cabrera-Gaytán DA, Niebla-Fuentes MD, Padilla-Velázquez R, ValleAlvarado G, Arriaga-Nieto L, Rojas-Mendoza T, et al. Association of pulmonary tuberculosis and HIV in the Mexican Institute of Social Security, 2006-2014. PLoS One. 2016;11(12):e0168559.

4. World Health Organization (WHO). Global Tuberculosis Report 2018 [Internet]. Genebra: WHO; 2017. [cited in 2019 Jul 11]. Available from: https://www.who.int/tb/publications/global_report/en/

5. Brasil. Ministério da Saúde. Boletim Epidemiológico Secretaria de Vigilância em Saúde. Coinfecção TB-HIV no Brasil: panorama epidemiológico e atividades colaborativas [Internet]. Brasília (DF): Ministério da Saude; 2017. [citado 2019 Jul 11]. Disponível em: http://www.aids.gov.br/pt-br/pub/2017/coinfeccao-tb-hiv-no-brasilpanorama-epidemiologico-e-atividades-colaborativas-2017

6. Sinshaw Y, Alemu S, Fekadu A, Gizachew M. Successful TB treatment outcome and its associated factors among TB/HIV co-infected patients attending Gondar University Referral Hospital, Northwest Ethiopia: an institution based cross-sectional study. BMC Infect Dis. 2017;17(1):132. [cited 2018 Sep 02]

7. World Health Organization (WHO). Companion handbook to the WHO guidelines for the programmatic management of drug-resistant tuberculosis [Internet]. Genebra: WHO; 2014 [cited 2019 Jul 11]. Available from: https://www.who.int/tb/publications/pmdt_companionhandbooken/

8. Mendes KD, Silveira RC, Galvão CM. Revisão integrativa: método de pesquisa para a incorporação de evidências na saúde e na enfermagem. Texto Contexto Enferm. 2008;17(4):758-64.

9. Moher D, Liberati A, Tetzlaff J, Altman DG; PRISMA Group. Preferred reporting items for systematic reviews and meta-analyses: the PRISMA statement. PLoS Med. 2009;6(7):e1000097.

10. Souza MT, Silva MD, Carvalho R. Integrative review: what is it? How to do it? einstein (Sao Paulo). 2010;8(1):102-6.

11. Ursi ES, Gavão CM. [Perioperative prevention of skin injury: an integrative literature review]. Rev LatAm Enfermagem. 2006;14(1):124-31.Portuguese.

12. Melnyk BM, Fineout-Overholt E. Evidence-based practice in nursing \& healthcare: A guide to best practice. Philadelphia: Lippincott Williams \& Wilkins; 2011.

13. de Kock L, Sy SK, Rosenkranz B, Diacon AH, Prescott K, Hernandez KR, et al. Pharmacokinetics of para-aminosalicylic acid in HIV-uninfected and HIV-coinfected tuberculosis patients receiving antiretroviral therapy, managed for multidrug-resistant and extensively drug-resistant tuberculosis. Antimicrob Agents Chemother. 2014;58(10):6242-50.

14. Fischl MA, Uttamchandani RB, Daikos GL, Poblete RB, Moreno JN, Reyes RR, et al. An outbreak of tuberculosis caused by multiple-drugresistant tubercle bacilli among patients with HIV infection. Ann Intern Med. 1992;117(3):177-83.

15. Campos PE, Suarez PG, Sanchez J, Zavala D, Arevalo J, Ticona E, et al. Multidrug-resistant Mycobacterium tuberculosis in HIV-infected persons, Peru. Emerg Infect Dis. 2003;9(12):1571-8.

16. Elmi OS, Hasan $H$, Abdullah S, Mat Jeab MZ, Bin Alwi Z, Naing NN. Multidrug-resistant tuberculosis and risk factors associated with its development: a retrospective study. J Infect Dev Ctries. 2015;9(10):1076-85.

17. Aaron L, Saadoun D, Calatroni I, Launay O, Mémain N, Vincent V, et al. Tuberculosis in HIV-infected patients: a comprehensive review. Clin Microbiol Infect. 2004;10(5):388-98.

18. Berhan A, Berhan Y, Yizengaw D. A meta-analysis of drug resistant tuberculosis in Sub-Saharan Africa: how strongly associated with previous treatment and HIV co-infection? Ethiop J Health Sci. 2013;23(3):271-82.
19. Janbaz KH, Qadir Ml, Ahmad B, Sarwar A, Yaqoob N, Masood Ml. Tuberculosis: burning issues: multidrug resistance and HIV-coinfection. Crit Rev Microbiol. 2012;38(4):267-75.

20. Munawwar A, Singh S. AIDS associated tuberculosis: a catastrophic collision to evade the host immune system. Tuberculosis (Edinb). 2012;92(5):384-7.

21. Vermund SH, Yamamoto N. Co-infection with human immunodeficiency virus and tuberculosis in Asia. Tuberculosis (Edinb). 2007;87(1 Suppl 1):S18-25.

22. Sacks LV, Pendle S, Orlovic D, Blumberg L, Constantinou C. A comparison of outbreak- and nonoutbreak-related multidrug-resistant tuberculosis among human immunodeficiency virus-infected patients in a South African hospital. Clin Infect Dis. 1999;29(1):96-101.

23. Bifani P, Mathema B, Kurepina N, Shashkina E, Bertout J, Blanchis AS, et al. The evolution of drug resistance in Mycobacterium tuberculosis: from a mono-rifampin-resistant cluster into increasingly multidrug-resistant variants in an HIV-seropositive population. J Infect Dis. 2008;198(1):90-4.

24. Chakraborty N, De C, Bhattacharyya S, Mukherjee A, Santra S, Banerjee D, et al. Drug susceptibility profile of Mycobacterium tuberculosis isolated from HIV infected and uninfected pulmonary tuberculosis patients in eastern India. Trans R Soc Trop Med Hyg. 2010;104(3):195-201.

25. Drobniewski FA, Balabanova YM, Ruddy MC, Graham C, Kuznetzov SI, Gusarova Gl, et al. Tuberculosis, HIV seroprevalence and intravenous drug abuse in prisoners. Eur Respir J. 2005;26(2):298-304.

26. Heysell SK, Ogarkov OB, Zhdanova S, Zorkaltseva E, Shugaeva S, Gratz $J$, et al. Undertreated HIV and drug-resistant tuberculosis at a referral hospital in Irkutsk, Siberia. Int J Tuberc Lung Dis. 2016;20(2):187-92.

27. Hom JK, Wang B, Chetty S, Giddy J, Mazibuko M, Allen J, et al. Drugresistant tuberculosis among HIV-infected patients starting antiretroviral therapy in Durban, South Africa. PLoS One. 2012;7(8):e43281.

28. Kim HR, Hwang SS, Kim HJ, Lee SM, Yoo CG, Kim YW, etal. Impact of extensive drug resistance on treatment outcomes in non-HIV-infected patients with multidrug-resistant tuberculosis. Clin Infect Dis. 2007;45(10):1290-5.

29. Mcllleron H, Rustomjee R, Vahedi M, Mthiyane T, Denti P, Connolly C, et al. Reduced antituberculosis drug concentrations in HIV-infected patients who are men or have low weight: implications for international dosing guidelines. Antimicrob Agents Chemother. 2012;56(6):3232-8.

30. Kawai V, Soto G, Gilman RH, Bautista CT, Caviedes L, Huaroto L, et al. Tuberculosis mortality, drug resistance, and infectiousness in patients with and without HIV infection in Peru. Am J Trop Med Hyg. 2006;75(6):1027-33.

31. Rockwood N, Sirgel F, Streicher E, Warren R, Meintjes G, Wilkinson RJ. Low frequency of acquired isoniazid and rifampicin resistance in rifampicinsusceptible pulmonary tuberculosis in a setting of high HIV-1 infection and tuberculosis coprevalence. J Infect Dis. 2017;216(6):632-40.

32. El Hamdouni M, Ahid S, Bourkadi JE, Benamor J, Hassar M, Cherrah $Y$. Incidence of adverse reactions caused by first-line anti-tuberculosis drugs and treatment outcome of pulmonary tuberculosis patients in Morocco. Infection. 2019.

33. Gualano G, Mencarini P, Musso M, Mosti S, Santangelo L, Murachelli S, et al. Putting in harm to cure: drug related adverse events do not affect outcome of patients receiving treatment for multidrug-resistant Tuberculosis. Experience from a tertiary hospital in Italy. PLoS One. 2019;14(2):e0212948.

34. Eshetie S, Gizachew M, Alebel A, van Soolingen D. Tuberculosis treatment outcomes in Ethiopia from 2003 to 2016, and impact of HIV co-infection and prior drug exposure: A systematic review and metaanalysis. PLoS One. 2018;13(3):e0194675.

35. Queiroz CA, Silvestre LR, Carmo TM, Andrade RD, Moura JP, Silva PG, et al. [AIDS-associated tuberculosis: an analysis of the prevalence of coinfection. Ciência ET Praxis. 2019;11(21):65-70. Portuguese. 